\title{
VARIABEL
}

e-ISSN: 2593-302X dan p-ISSN: 2599-3038

This work is licensed under

\section{Profile of Mathematics Education Students' Understanding with Moderate Mathematics Ability in the Aspect of Dissection of Group}

\author{
Jafar $^{1}$, I Ketut Budayasa ${ }^{2}$, Dwi Juniati ${ }^{2}$ \\ Department of Mathematics Education, FKIP Halu Oleo University, Bumi Tri Dharma \\ Anduonohu Kendari ${ }^{1}$ \\ State University of Surabaya, Ketintang Campus Building K-9 Surabaya ${ }^{2}$ \\ jafar665@gmail.com ${ }^{1}$,dwi_juniati@yahoo.com ${ }^{2}$
}

\section{Keywords :}

dissection of concept, group,

understanding profile

\begin{abstract}
One aspect that can be used to identify the completeness of the students' understanding to a concept is by doing dissection to the concept. This is a case study on the students of mathematics education who had moderate ability, and the objective was to obtain the profile of students' understanding towards group concept on the aspect of dissection. The students' understanding to the concept is needed very much to build foundation for understanding to other concepts in abstract algebra. Based on the result of the study, it was obtained that although the dominant elements that build the concept of group can be identified well but qualitatively the understanding of the students who have moderate ability in mathematics to the group concept is still weak. One of the causes is the mathematics logic as an instrument in managing information in group concept is not used accurately and tightly.
\end{abstract}

\section{INTRODUCTION}

One widely accepted mathematical idea in mathematics learning is that students must understand mathematics (Hiebert and Carpenter in Godino, 1996). Meanwhile, interest in the teaching and learning of mathematics with understanding is seen important, as demonstrated by curriculum reformation in various countries (Pirie and Kieren in Godino, 1996). It shows that in mathematics learning, understanding of mathematical concepts is a very important component.

On the other hand, mathematical concepts have abstract characters. Even most of these concepts are 'very' abstract and therefore it requires high knowledge activity in learning it (Okur et al., 2011).

The abstract algebra as one of the mathematics branches contains concepts that have abstract structures (Findell, 2001). Abstract algebra is a generalization of school algebra in which the values substitution of the variables is a variety of mathematical objects (Findell, 2001), and the expressions are also built with general operations. Objects in abstract algebra not only relate to mathematical objects that have been commonly known and used in school algebra. Therefore, objects in abstract algebra seem more abstract than the mathematical objects studied in school algebra. Therefore, to understand concepts in abstract algebra requires a high level of knowledge, and studying the 
relationships between objects in abstract algebra requires strong reasoning. It is implied to the students' ability to understand concepts in abstract algebra. Abstract algebra teachers recognize that most students view that abstract algebraic material is very hard to understand, and they also find difficulties in communicating ideas in abstract algebra (Hazzan, 1999). On the other hand, teachers also realize the importance of learning abstract algebra. Therefore the learning process should be directed to learning with understanding.

Several studies have shown that students' understanding of concepts in abstract algebra is less satisfactory (Findell, 2001). Harel suggests several factors that make abstract algebra difficult for students. First, abstract algebra concepts are very abstract structures that serve as categories for a wide range and with diverse examples. Objects are determined by their characters, so it makes students difficult to understand them. Second, most examples that explain the concepts are not familiar to students. Third, most students have not felt comfortable with proof by axiomatic methods (Findell, 2001). In addition, empirically based on data from 2005-2010, the results of midterms (UTS) or semester final examination (UAS) of Mathematics Education students of Halu Oleo University in abstract algebra courses are still relatively low, as a result of students' habits in learning mathematics that rely solely on the aspects of calculation ability, such as arithmetic, school algebra (low algebra), and calculus, formed on learning in primary and secondary education (Jafar, 2013).

Kaput (2005) states that algebra has been traditionally understood and studied as a set of procedures that is disconnected both from other mathematical knowledge and from real life. It is understandable that algebraic learning (including abstract algebra) has not been able to approach algebraic concepts with students' interaction in everyday life. Meanwhile, Susanto (2011) found that the lecturers have not been optimal in paying attention the understanding of the concept and understanding in solving the problem of proof in the learning process of abstract algebra.

The above description indicates that there are still problems with the understanding of students in learning abstract algebra concepts. Of course, one strategy or approach that can be done is to improve the learning process of abstract algebra. The purpose of this paper is to reveal the understanding profile of mathematics students who are capable in moderate mathematics on group concepts in the aspects of dissection. This profile is needed to be the foundation for improving the abstract algebra learning process. Although in this paper the study is limited to students' understanding of group concepts, but it can be developed and exploited as necessary against the other concepts.

\section{Students' Understanding on a Concept}

Etymologically, understanding is a psychological process related to an abstract or physical object, such as a situation, person, or message in which one can think and use concepts to describe the object. Suzana (2006) says that understanding is the ability to explain a situation or an action. Understanding is also seen as a mental experience gained from the activity to understand a concept. According to Sierpinska (Godino, 1996), understanding is the mental experience that connects one object with other ones.

An individual's understanding of a concept is the result of the individual's mental activity in understanding the concept. Someone understands a concept because he has done thinking activity about the concept. Skemp (1982) asserts that "To understand something means to assimilate it into an appropriate schema." It implies that someone is said "understanding something" when there has been integration of new information with the scheme that the person has. It can be said that understanding is concerned with a person's ability to integrate 'new' information through the accommodation and assimilation processes into the scheme that the person has before to form a new scheme.

Hiebert and Carpenter (1992) suggest that what is meant by a person's understanding of a concept is a condition that describes suitability between mental representation or an internal representation of the concept and the network of representations that the person has. Furthermore Barmby et al. (2007) suggest that the quality of understanding is determined by the number of information networks and strong relationships (interconnection) between sub-networks owned. Meanwhile, Jafar (2013) suggests 
that a concept can be completely understood by an individual if the individual can build linking networks as many as possible between the attributes contained in the concept with the scheme owned, and the strong linkage with the network that has been owned by the individual.

A person's understanding toward a concept is not easy to observe precisely, and can only be known through interviews. Jafar (2013) in elaborating the understanding quality of a concept proposed by Barmby above, formulates several categories that enable us to detect the quality of one's understanding on a concept as follows.1) Complete disclosure of the concept with the own words. 2) Identification of all components contained in a concept. 3) Explanation of each component in concept accompanied by its strengthening. 4) Connection between components in a concept. 5) The use of a concept to solve related problems.

One of the concepts in abstract algebra is group. The group is the earliest learned basic structures in abstract algebra (Herstein, 1975), and become the main foundation for building other structures, such as ring, field, and vector space, ect.

Conceptually, a group is a structure constructed by a non-empty set and a binary operation, called a product, and has characters: closed, associative, having identity elements, and there is inverse of each element. Herstein (1975) constructs the definition of the group as follows.

A non-empty set and a binary operation, called the product, is symbolized $\cdot$, it is said to form a group, if

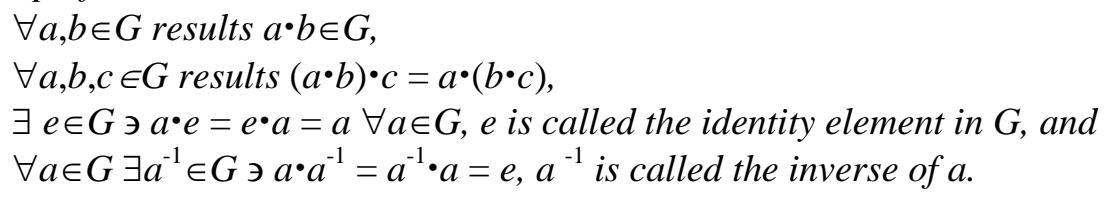

Remembering that the group concept is a structure that becomes the foundation or base to build other structures, then the incomplete students' understanding of the concept will be implicated in the onset of their difficulties in understanding the concepts or other structures.

According to Jafar (2013), a complete understanding on the group concept can be obtained when students have had the following things: First, the ability to identify all components that build the group along with the characters; Second, the ability to find linkages between components; Third, the ability to find easily, either as a representation of each component of a group component, or as a representation for the group concept; Fourth, the ability to construct concepts that are the opposite of group concepts, and easily to find examples; Fifth, the ability to use group concepts to solve related problems. In detail, Jafar (2014) suggests there are four aspects that can be used to detect the quality of students' understanding on the group concept, namely general explanation, dissection, dependability, and affirmation.

\section{Students' Understanding on Group Concepts}

It has been argued that one aspect to detect the quality of student's ability to understand the group concept completely is dissection concept. The ability of students to perform group concept dissection is seen from two things, namely the ability to identify all components of the group concept, and the ability to explain in detail the meaning of each component. In detail, this aspect can be reviewed from five activities: identifying the basic elements that build the group concept, explaining the meaning of each basic element, identifying the characters of each basic element that build the group concept, explaining the meaning of each character of each basic element, identifying the essential characters of the group, and explaining the meaning of each character.

\section{METHOD}

This research was qualitative exploratory study. The subject was a student at the Department of Mathematics Education FKIP UHO who had the moderate mathematics ability, by the following 
criteria: 1) having studied the group concept, 2) having the math skills test scores (TKM), which is in the range 60 to 75 , and 3 ) having $2.50 \leq \mathrm{GPA} \leq 3.00$.

The data collection of this research was done by interview method in depth, overt, and unstructured. For the purpose of obtaining valid and credible data, triangulation was performed, with a type of time triangulation. Data processing research was done by qualitative data analysis method Miles and Huberman model through stages: 1) data reduction, 2) data presentation, and 3) drawing conclusion.

\section{RESULTS AND DISCUSSIONS}

In this section we will present an understanding profile of mathematics education students who have moderate math skills (AI) on group concepts on aspects of group concept dissection. The aspect consists of identifying, and explaining in detail all components of the group concept. This profile is obtained through analysis of data in-depth interviews that the researcher did to AI.

\section{Identifying Basic Elements that Builder of Group}

This activity was related to AI's ability to find the components that became the foundation or foundation for building a group. AI said that the elements constructing group concepts were nonempty sets and binary operations. Then the nature of set was not empty. Thus it can be seen that AI could identify the elements of group builder and also the dominant nature of set that built the group concept.

\section{Explaining the Understanding of Any Element that Builds Group}

This activity was related to AI's explanation of the meaning of every basic component that built a group, namely set and binary operations in accordance with its understanding.

1. Set

AI understands set as a set of clearly-defined objects. To provide affirmation about set, AI gave an example which was a representation of the concept of set. The set of natural numbers was one example of a set since this set began with the numbers 1 and so on, and -3 was not a natural number. Furthermore, about not the set, AI understood it as a collection of objects that could not be clearly defined, reinforced by example a collection of beautiful women because there was no measurement to determine a woman was beautiful. Thus it can be put forward the following things. First, AI's explanation about the meaning of set had corresponded to the conceptual meaning of set. Second, when giving a set example, AI referred to the meaning of set, as well as the reasons put forward for the selection of such examples. Third, when giving the definition of a non-set concept, AI referred to the essence of set definition as it is proposed. Fourth, examples of non-set objects given by AI appeared to correspond to the definition of objects that were not binary operations as he proposed, but the reason for the selection did not refer to the essence of the membership requirement of a set. In this case substantial inadequacy was still found. This inaccuracy showed that AI had not explicitly distinguished between the membership requirements of a set and the inherent natures or characters of a set member.

From above description we can know that the AI understanding of sets concept was already quite strong, although it was still found inaccuracy when AI argued the sample selection of non set.

\section{Binary Operation}

AI understood binary operations in a set as a mapping that mapped the consecutive pairs of elements in a given set then mapped exactly one to the set itself. To reinforce the explanation, then AI gave an example of binary operation in a set. The multiplication operation on the set of integers was one example of binary operation since when taking integers 2 and 3, then multiplied it could be paired exactly one with an integer 6 . Next, about not a binary operation on a set, AI said that when taking any the pair of elements in its set then operated by binary operations mapped to one element that was not a member of the set. Then AI gave an example $A=\{-1,1\}$ with the addition operation because if -1 and -1 were summed, then the result was -2 which was not an element in $A$. From here it can be put 
forward the following. First, AI's explanation about binary operations had led to a conceptual binary operation definition. Second, when giving an example of binary operations, AI referred to the definition of binary operations he proposed, as well as the reasons in the selection of examples. Third, when proposing the definition of a concept that was not a binary operation, AI only viewed the dominant element in terms of its own binary operation, namely the result of association of two elements in the set given to the set, and did not refer to the essence of binary operations as a mapping. Based on the explanation, it can be seen that substantially, there was still incompleteness of AI understanding on binary operations. This incompleteness illustrated further that AI's understanding of the concept of mapping was not yet strong. Fourth, examples of objects that were not binary operations given by AI appeared to correspond to the definition of objects that were not binary operations as he expressed, as well as the reasons for the selection. Thus, it can be seen that AI's understanding of binary operations is not yet strong enough.

\section{Identifying the Properties of Builders of Group}

Concretely, the natures of the group concept builder are the non-empty property of the set, while the binary operation natures are directly attached to the definition of the binary operation concept itself.

About the non-empty set, AI understood it as a set that had members. To affirm the explanation, then AI gave an example, among others the set of integers, since 1 was one of its members. Furthermore, about the empty set, AI said that it was the set did not have members. Then AI exemplified the set of natural numbers between -5 and -10 since there was no member qualified for membership. It can be put forward the following things. First, the definition of the non-empty set proposed by AI had corresponded to this definition theoretically. Second, when exemplifying a non-empty set, it appeared that AI referred to the definition of a non-empty set as it was proposed. It was reinforced also by reason of the sample selection. Third, when proposing the definition of the empty set concept, it appeared that AI referred to the definition of a non-empty set. Fourth, the sample selection for the empty set referred to the definition of the empty set that he expressed before although the reason for selecting the example was not explicitly. Based on the four reasons it can be seen that the understanding of AI to the set of non-empty is quite strong.

\section{Identifying the Essential Properties of Group}

In the phase of identifying the essential natures of the group concept, AI said that the essential natures of the concept of four groups were closed, associative, there was an element of identity, and every element had an inverse. It showed that concretely AI had identified all the essential characteristics of the group concept, as the dominant part of the group concept.

\section{Explaining the Definition of each Essential Properties of the Group}

This activity is related to the ability of AI to explain each of the essential property of the group in accordance with his understanding.

\section{The Closed Property}

When describing the definition of closed property, AI emphasized the existence of any element in a given set in which, if operated by a given binary operation, it produced elements in the set. To affirm the explanation, then AI gave an example, the addition operation on a set of integers because when adding the two integers then the result was an integer as well. Furthermore, on binary operations that did not meet closed property, AI said that when taking any two elements in the set and operated, the result was an element that was not in the set. Then AI exemplified substraction operation on the set of natural numbers because if taking the natural number 1 and 2, then substracted, $1-2=-1$, whereas -1 was not a natural number. In this regard, it can be summarized as follows. First, the definition that had been pointed out had led to a conceptual understanding. Second, the sample selection of binary operations in a set that met the closed nature referred to the definition of this nature as it was said, similarly, the reason for choosing the examples. Third, when proposing the definition of the binary operation that did not meet the closed property, AI only focused on the dominant elements in the definition of this property, namely the result of two elements operation in the set given to produce an 
element that was not in the set, and did not refer to the essence of this property definition conceptually implying that there was no binary operations that was not closed. In this case, AI did not relate this nature to the essence of binary operation definition itself. It was also affirmed by the sample selection on this subject, and the reason for it. It showed that substantially, there was an incomplete understanding of AI on sets and binary operations that did not meet the closed property. These three points above indicated that AI's understanding on binary operations that met the closed property was still incomplete.

\section{Associative Property}

AI 's explanation on the definition of associative nature was that AI emphasized the action of taking any three elements $a, b, c$, then $a$ was operated with $b$, then the result was operated with $c$, it would be equal to operating first $b$ with $c$, then the result was operated with $a$. As an affirmation of this explanation, AI gave an example that met this property, one of which was addition operation on the set of integers. However, in this example it appeared that AI had little difficulty in constructing the argument because the elements in the set were 'unlimited'. AI only gave an example by selecting 1,2 , and 3 then finding that $(1+2)+3=1+(2+3)$ although AI recognized that it should test all possibilities. However, since the elements were 'unlimited', then according to AI, it was sufficiently defined to be ' $a+(b+c)=(a+b)+c$ '. Furthermore, about binary operations that did not meet associative property, AI said that when two grouping operations were created, the results were not the same. Then AI exemplified binary operations $*$ on $\{0,1\}$ where $1 * 1=1,1 * 0=2,0 * 1=0$, and $0 * 0=$ 1 , because if taking 1,0 , and $0,1 *(0 * 0)=1$, whereas $(1 * 0)^{*} 0=2 * 0$ was undefined.

In this regard, it can be argued as follows. First, the proposed definition did not lead to a conceptual understanding. The AI's explanation led to a procedure to prove this property. This is affirmed by the selection of binary operation examples in a set that met the closed property, along with the reasons for the election. The phrase '... taking any ...' indicated it. Second, when presenting the example of sets and binary operations that met this property, AI referred to the definition of this property as it is proposed. It was illustrated by the argument of sample selection that he was constructing. Third, when proposing the definition of binary operations that did not meet associative property, AI only focused on the dominant element in the definition of this property namely the multiplication result of the three elements in the set given in different groupings gave unequal results, and of course it did not refer to the essence of this property conceptually which required that the similarity of the results of the three elements operation in their set in different groupings would apply to all elements in the set. Thus, it can be seen that substantially there was an incompleteness of AI's understanding on this associative property. This incompleteness had the potential to understand sets and binary operations that did not meet this property on the term "if all three element choices in a given set after being operated in different groupings always give different results". Fourth, an example of a set and a binary operation that did not fulfill this property referred to the definition constructed, and so did the reason for it. These four things indicated that AI's understanding on binary operations that met associative properties was still not solid.

\section{The Existence of Identity Element}

AI's explanation on the nature of the identity element existence did not lead to a conceptual understanding. AI said that, "every $x$ element in $A$ then there was $e$ in $A$, such that $a$ was operated with $e$ equaled to $e$ operated with $a$, equal to $a$. $e$ was called the identity element in $A$ ". As the affirmation of his explanation of this property, AI gave an example, one of which was a sum operation on a set of integers, in which in this case the identity element was 0 since according to AI that each element in $A$ when summed with 0 produced that element. Furthermore, about binary operations that did not meet the property of the identity element, AI said that there was $b$ an element in the set in which, when operated with $e$, it did not produce $b$. About sets and binary operations that did not meet the nature of identity elements, AI exemplified the set of integers without 0 by addition operations, in which I this case AI considered the example of a set and a binary operation that had an identity element, then he made it as an example that did not meet this nature after removing the identity element. 
Therefore, relating to the solidity of AI's understanding on the property of identity elements, the following points are expressed. First, AI's explanation about the definition of this property was inconsistent with theoretical definition since it was as if the identity element attached to each element. The statement put forward by AI began the definition of this nature, namely "every element [a] in set A, for example there is an e member in the set ..." indicating this. This kind of understanding was not strong enough to use a concept in solving related problems, especially in proving the theorems related to the use of this nature. Second, when thinking an example of a set and a binary operation that met the property of an identity element, AI decided the set first before determining its operation. The selection of such examples 'as if' did not refer to the definition of a binary operation that met the property in accordance with what had been said. In this case, when constructing the argument that in the set and the binary operation he chose had an identity element, AI tried to directly guess the identity element, then proved that it met the property. Third, when explaining the definition of set and binary operations that did not have an identity element, AI focused only on the dominant elements, namely the results of operation $b$ with $e$ which did not produce $b$ itself. Implicitly, what AI understood about this showed a contradiction. On one hand, what was defined was the definition of set and binary operations that did not have an identity element, while on the other hand, the statement that was said involved identity elements. Thus, in this context there was a partial substantial disclosure of AI's understanding on the definition of sets and binary operations that did not meet this property. Fourth, when giving examples of sets and binary operations that did not meet this property, AI did it by first thinking of the set and binary operations that had an identity element, then he used it as an example that did not meet this property after removing its identity elements even though AI realized that it resulted the set and binary operation referred not to meet the closed property. It provided an illustration that the selection of set sample and binary operations that did not meet this property did not refer to the definition of the set and binary operations that did not meet this property as it was proposed. In this context it can be viewed as an AI's inconsistency when choosing the example as well as the reason for its election.

\section{Existence of Inverse of Each Element}

When explaining the nature of the inverse of each element, AI said, "for every $a$ element $A$, then there is $a$ inverse which when $a$ is operated with $a$ inverse, equal to $a$ inverse operated with $a$, equal to $e$, in which $e$ is an identity element in set $A$ ". As an affirmation of his explanation about the property of inverse of each element in the set given in accordance with this understanding, AI exemplified the set of integers by addition operation, because according to him, when $a$ was summed with $a$ inverse, the result was 0 , and 0 was the element of identity. This reason was affirmed by if choosing $a=2$, then the inverse of $a$ was -2 . Another example proposed by AI was the set of non-zero rational numbers with multiplication operation since according to AI, by taking any rational number, for example 2 then there was $1 / 2$ rational number so $2 \times 1 / 2$ equaled to its identity element, namely 1 . Similarly, if the rational number was $a$, then $a$ inverse was $1 / a$. Furthermore, regarding binary operations that did not meet the inverse of each element, AI said that it meant there was $y$ of an element in its set in which, when operated with its $y$-bar (that was, the inverse of $y$ ) it did not result an identity element. About binary sets and operations that did not meet the property of the identity element, AI exemplified the set of integers without -2 with addition operation. In this case AI considered the examples of a set and binary operations of which each element had an inverse, then he made it as an example that did not meet this nature after removing one of its elements, namely -2 . From this example, AI argued that 2 did not have inverse since the inverse of 2 should be -2 , but -2 had been removed.

Therefore, relating to the solidity of AI's understanding on the property of the inverse of each element, the following points are made. First, the AI's explanation about the definition of this property had corresponded to the theoretical definition. Second, when thinking an example of a set and a binary operation that met this property, AI decided the set first before determining its operation. However, it appeared that the selection of the example referred to the definition of a binary operation that met the propery in accordance with what had been said. However, when constructing arguments that each element in the set and binary operations chosen had an inverse, it had a logical inconsistency, so it gave the impression that there was inefficiency in the argument. Third, when explaining the definition of set and binary operations that not all elements had an inverse, AI only focused on the dominant elements, namely the operation result of $y$ with its inverse which did not result an element of identity. 
Implicitly, what the AI understood about it showed a contradiction. On one hand, what was formulated was the definition of sets and binary operations that not all elements had inverse, while on the other hand, the statement that he said involved inverse of the elements. Thus, in this context, logically, there was a partial substantial disclosure of AI's understanding on the definition of sets and binary operations that did not meet this nature. Fourth, when giving examples of sets and binary operations that did not meet this property, AI did it by first thinking of the set and binary operations that each element had an inverse, then using it as an example that did not meet this property after removing one of its elements. It provided an illustration that the selection of set sample and binary operations that did not meet this nature referred to the definition of sets and binary operations that did not meet this property as what it was expressed although the reasons did not refer to the intended definition. In this context it could be viewed as an inconsistency of AI when establishing the reason for selection of the example. The four points above illustrate that AI's understanding of the nature of the inverse of each element was not solid enough.

Based on detailed descriptions in the data analysis and discussion section relating to AI's activities in conducting group concept dissection, the profile is obtained, and schematically presented in Figure 1. 


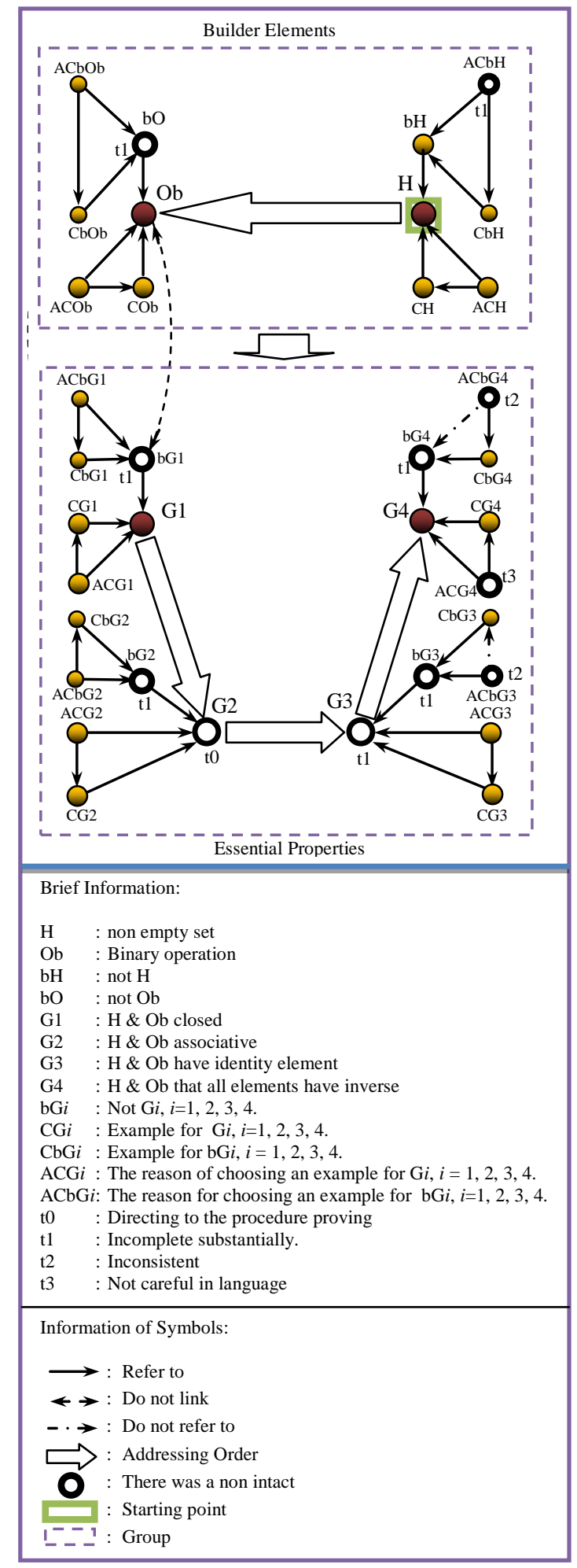

Fig 1. Understanding Profile of Research Subjects on the Group Concept on Dissection Aspects

\section{CONCLUSION AND SUGGESTION}

As a conclusion of this study, some matters are raised as the following:

a. Quantitatively, the subject of this study has been able to identify all the dominant components in group concepts, non-empty sets, binary operations, and essential natures or axioms of group.

b. Qualitatively, the understanding of the research subject to the group concept is not strong enough. It is based on the following reasons. First, not all of the group concept component definitions that 
he presented lead to definition theoretically. Second, in general, AI's explanation on the definition of each group concept component only focuses on the dominant elements. It is illustrated when AI explained the definition of the object that was not a group component, such as the object that was not a binary operation, sets and binary operations that were not closed, and so on. Third, most of the arguments developed by the research subject related to the sample selection or not a sample of each component that builds the group, logically, and it does not refer to the definition of the components.

\section{REFERENCES}

Barmby, P., Harries, T., Higgins, S., \& Suggate J. (2007). How Can We Assess Mathematical Understanding?. Proceedings of The $31^{\text {st }}$ Conference of The International Group for The Psychology of Mathematics Education, 2, 41-48.

Findell, B.R. (2001). Learning and Understanding in Abstract Algebra. Doctoral Dissertation. University of New Hampshire. (Unpublished).

Godino, J. D. (1996). Mathematical Concept, Their meanings, and Understanding. Proceedings of XX Conference of The International Group for The Psychology of Mathematics Education, 2, 417425 .

Hazzan, O. (1999). Reducing Abstraction Level when Learning Abstract Algebra Concepts. Educational Studies in Mathematics, 40, 71-90.

Herstein, I. N. (1975). Topics in Algebra. Second Edition. New York: John Wiley \& sons, Inc.

Hiebert, J., \& Carpenter, T. P. (1992). Handbook of Research on Mathematics Teaching and Learning: Learning and Teaching with Understanding. New York: MacMillan.

Jafar. (2013). Membangun Pemahaman yang Lengkap (Completely Understanding) dalam Pembelajaran Konsep Grup. Prosiding Konferensi Nasional Pendidikan Matematika (KNPM) V, Malang Indonesia, 87-95.

Jafar. (2014). Aspek-Aspek untuk Mengidentifikasi Kekuatan Jaringan Pemahaman Mahasiswa terhadap Konsep Grup. Makalah. Disajikan pada Seminar Nasional Matematika, 1 Februari, FMIPA UI Jakarta.

Kaput, J. J. (2005). Teaching and Learning a New Algebra with Understanding. Dartmouth: University of Massachusetts.

Okur, M., Dìkìcì, R., Sanalan, V. A., \& Tatar, E. (2011). Computer Applications in Teaching Abstract Algebra. International Journal of Applied Science and Technology, 1(1), 20-27.

Skemp, R. R. (1982). The Psychology of Learning Mathematics. New York: Penguin Books.

Susanto, H. A. (2011). Pemahaman Mahasiswa dalam Pemecahan Masalah Pembuktian pada Konsep Grup Berdasarkan Gaya Kognitif. Disertasi Doktoral. Surabaya: Universitas Negeri Surabaya. (Unpublished).

Suzana, Y. (2006). Meningkatkan Kemampuan Pemahaman dan Penalaran Matematika Siswa SMU Melalui Pembelajaran dengan Pendekatan Metakognitif. Tesis. Bandung: Universitas Pendidikan Indonesia. (Unpublished). 